\title{
The Response to Acoustic Stimulation and the Changes in Brain Amine Levels after Repeated Administration of $\beta$-Phenylethylamine in Rats
}

\author{
Mitsuhiko Yamada, Yuji Kiuchi, Miyuki Hashimoto, \\ Katsuji Oguchi and Hajime Yasuhara \\ Department of Pharmacology, School of Medicine, Showa University, \\ 1-5-8 Halanodai, Shinagawa-ku, Tokyo 142, Japan \\ Received November 30, 1990 Accepted March 11, 1991
}

\begin{abstract}
Reverse tolerance to stereotyped behavior was induced after repeated administration of $\beta$-phenylethylamine (PEA) $(50 \mathrm{mg} / \mathrm{kg}$, i.p., daily for 10 days) in rats. The reverse tolerance was maintained for at least 4 weeks after the last administration. We studied the effects of acoustic stimulation on locomotor activity 2 days and 4 weeks after withdrawal from PEA and measured the changes in brain monoamine levels 4 weeks after the withdrawal. Locomotor activity during acoustic stimulation was increased in the saline treated group, and this response was unaffected after repeated PEA treatment. Four weeks after withdrawal, significant increases in noradrenaline levels in the cerebral cortex and decreases in 5-hydroxytryptamine levels in the hypothalamus were found. The effects of acoustic stimulation on locomotor activity and the changes in brain monoamine levels were different from those of methamphetamine treatment obtained in our previous study. In conclusion, it may be suggested that the response to acoustic stimulation after repeated PEA administration in rats cannot be a model for abnormal responsiveness to environmental stimulation that is observed in chronic paranoid schizophrenics.
\end{abstract}

$\beta$-Phenylethylamine (PEA) is a trace amine which is naturally present in the mammalian brain (1). PEA structurally resembles amphetamine (AMPH) and possesses similar pharmacological properties, which include its abilities to cause an increase in spontaneous motor activity $(2-4)$, appearance of stereotyped behaviors $(2,3,5)$ and decrease in food and water intake $(3,6)$. However, the physiological roles of PEA are not as fully understood.

After the repeated administration of PEA to rats, augmented stereotyped behavior is observed $(5,7,8)$. This phenomenon is also observed after repeated administration of AMPH (9) or methamphetamine (MAP) (10 -
12) and is called "reverse tolerance". It is proposed that the reverse tolerance to stercotyped behavior in animals may closely resemble AMPH psychosis in man, because of the similarity between the behavioral sensitization and relapse in psychosis $(13,14)$; and the stereotyped behavior observed in animals treated chronically with PEA has been proposed as a pharmacological model for schizophrenia $(5,7,15)$.

It is well-known that responses to environmental stimulation are abnormal in chronic paranoid schizophrenics $(16,17)$. In this study, we induced reverse tolerance to stereotyped behavior by repeated PEA administration in 
rats. Then we investigated the effects of acoustic stimulation on locomotor activity 2 days and 4 weeks after drug withdrawal and measured the levels of brain monoamines and their major metabolites 4 weeks after the withdrawal.

\section{MATERIALS AND METHODS}

\section{Animals}

Male Wistar rats aged 7 weeks and weighing $200-250 \mathrm{~g}$ at the beginning of this study were used. The rats were housed in groups of six and had continuous access to food and water except during the experimental sessions. Lighting was maintained on a 12-hour light/dark cycle under standard laboratory conditions.

\section{Groups and schedule}

The rats were divided into 4 groups. In each group, reverse tolerance to stereotyped behavior was induced. In the first group, we confirmed that reverse tolerance to stereotyped behavior was maintained at least for 4 weeks. In the second and the third groups, we studied the locomotor activity response to acoustic stimulation 2 days and 4 weeks after withdrawal, respectively. In the fourth group, the contents of brain monoamines and their metabolites were measured 4 weeks after withdrawal.

\section{Induction of reverse tolerance}

For inducing reverse tolerance to stereotyped behavior, PEA $(50 \mathrm{mg} / \mathrm{kg}$, i.p. $)$ or saline as a control was administered daily for ten days. To confirm the induction of reverse tolerance, stereotyped behavior was rated every $3 \mathrm{~min}$ for $45 \mathrm{~min}$ after each treatment on the first, second, third, fifth and tenth day. Animals were rated according to Borison et al. (5) with some modifications as follows: (0) inactive, lying down; 1) moving about cage, sniffing, rearing; 2) repetitive exploration of the cage with hyperactivity; 3) occasional sideto-side head bobbing, remains in one location; 4) continuous head bobbing, remains in one location for over five min. At least 2 hours prior to the observation, the rats were placed individually in the experimental cages, which were the standard wire mesh type, and allowed to adapt to the observation room. The same dose of PEA $(50 \mathrm{mg} / \mathrm{kg}$, i.p.) was given 4 weeks after withdrawal, and stereotyped behavior was scored again.

\section{Response to acoustic stimulation}

After the 2-hour adaptation to the cxperimental room which could be sealed off from outside noises and other disturbances, the behavioral tests were carried out. We used an open field apparatus (18) that consisted of a circular floor $(60 \mathrm{~cm}$ diameter $)$ and a surrounding wall expanding towards the upper brim $(60 \mathrm{~cm}$ high and $90 \mathrm{~cm}$ in diameter at the top). The floor was divided by black lines into 13 units of approximately equal size, and locomotor activity was counted individually for 12 min and expressed as the number of lines crossed by the rat per min. Between 4 to 5 $\min$ and between 9 to $10 \mathrm{~min}$, acoustic stimulation (peak frequency: $4 \mathrm{kHz}$, mean intensity of 3 points on the floor: $95.9 \mathrm{db}$ ) was given by a buzzer located one meter above the floor. Statistical evaluations were determined by the Mann-Whitney $U$-test.

\section{Contents of brain amines and their metabolites}

The contents of noradrenaline (NA), 3methoxy-4-hydroxyphenylglychol (MHPG), dopamine (DA), 3,4-dihydroxyphenylacetic acid (DOPAC), 5-hydroxytryptamine (5-HT), 5-hydroxyindoleacetic acid (5-HIAA) and homovanillic acid (HVA) were measured using HPLC-ECD $(19,20)$. The rats were sacrificed by decapitation, and the whole brains were removed immediately, placed on ice and dissected into seven regions (21). The cerebral cortex, midbrain and thalamus, hypothalamus, and striatum were used for the measurements. Each region was homogenized in $0.12 \mathrm{M}$ sodium acetate buffer ( $\mathrm{pH} 5.0)$ and then centrifuged at $15,000 \times g$ for $20 \mathrm{~min}$ at $4^{\circ} \mathrm{C}$. The supernatant was processed for enzymatic hydrolysis with sulfatase. After another centrifugation, the supernatant was washed with 
chloroform and then analyzed. Statistical evaluations were performed by Student's $t$-test.

\section{RESULTS}

\section{Induction of reverse tolerance}

Reverse tolerance to stereotyped behavior was induced after repeated PEA administration in rats. The reverse tolerance was maintained for at least 4 weeks after the last administration (data not shown).

\section{Response to acoustic stimulation}

Figure 1 shows the effects of acoustic stimulation on locomotor activity 2 days and 4 weeks after the last drug administration. As shown in Fig. 1a, 2 days after withdrawal, relatively high activity was observed during acoustic stimulation ( 15 counts at 4 to $5 \mathrm{~min}$ ), but the activity was completely suppressed after the acoustic stimulation. The same effects were observed 4 weeks after the withdrawal of PEA (Fig. 1b). However, there was no difference in the response between the PEA-treated group and the saline-treated group.

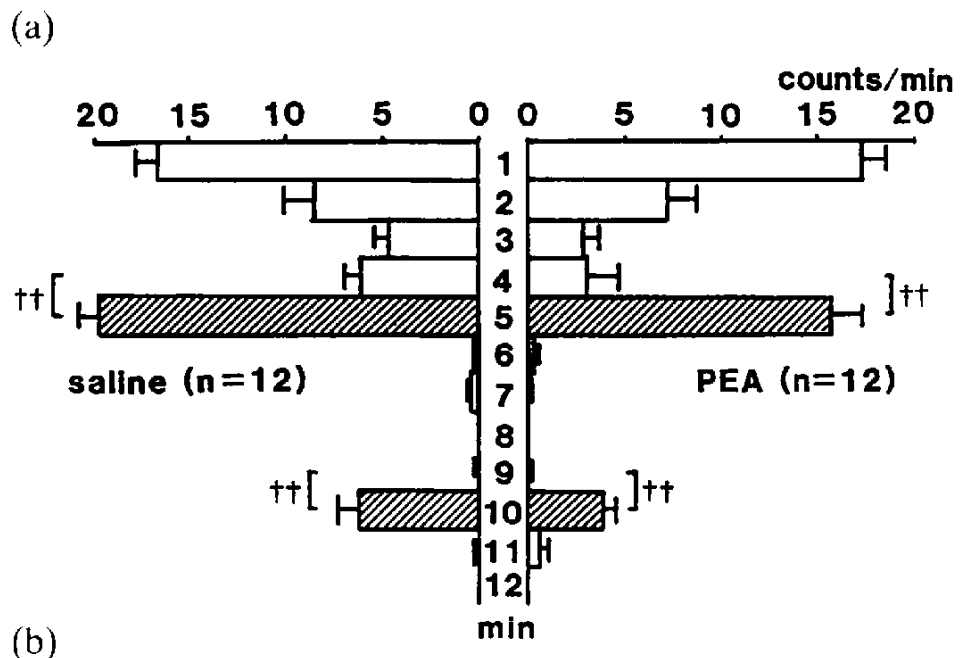

(b)

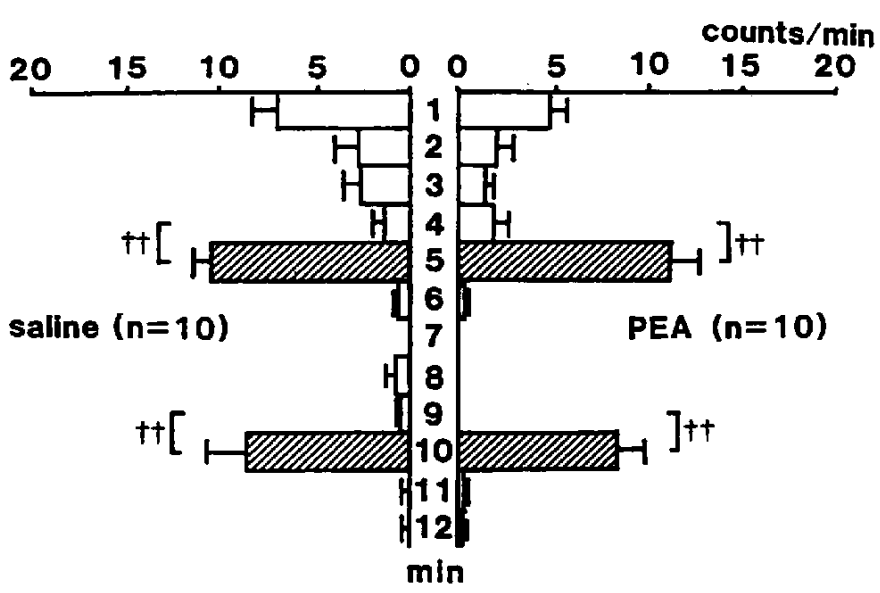

Fig. 1. Response to the acoustic stimuration was examined 2 days (a) and 4 weeks (b) after the repeated PEA administration. Locomotor activity was counted individually for $12 \mathrm{~min}$ using an open ficld apparatus. Between 4 to $5 \mathrm{~min}$ and between 9 to $10 \mathrm{~min}$ acoustic stimulation was given. VIIIIS: with or $\square$ : without acoustic stimulation. Values are mean \pm S.E.M. ${ }^{H}: \mathrm{P}<0.01$ (Mann-Whitney U-test). 
Table 1. Contents of brain amines and their metabolites 4 weeks after PEA withdrawal

\begin{tabular}{|c|c|c|c|c|c|c|c|}
\hline & NA & MHPG & DA & DOPAC & HVA & $5-\mathrm{HT}$ & 5-HIAA \\
\hline \multicolumn{8}{|c|}{ Ccrebral cortex } \\
\hline saline & $265 \pm 18$ & $101 \pm 60$ & $332 \pm 41$ & $173 \pm 13$ & $125 \pm 60$ & $224 \pm 26$ & $248 \pm 15$ \\
\hline PEA & $322 \pm 02^{*}$ & $114 \pm 70$ & $347 \pm 58$ & $186 \pm 18$ & $185 \pm 10$ & $212 \pm 15$ & $245 \pm 14$ \\
\hline \multicolumn{8}{|c|}{ Midbrain and thalamus } \\
\hline saline & $299 \pm 17$ & $121 \pm 10$ & $123 \pm 22$ & $75 \pm 06$ & $67 \pm 12$ & $390 \pm 45$ & $229 \pm 24$ \\
\hline PEA & $339 \pm 10$ & $124 \pm 80$ & $147 \pm 33$ & $86 \pm 10$ & $78 \pm 50$ & $377 \pm 45$ & $252+21$ \\
\hline \multicolumn{8}{|c|}{ Hypothalamus } \\
\hline saline & $1059 \pm 160$ & $154 \pm 10$ & $422 \pm 42$ & $314 \pm 36$ & $298 \pm 45$ & $482 \pm 27$ & $254 \pm 36$ \\
\hline PEA & $1069 \pm 128$ & $139 \pm 11$ & $472 \pm 48$ & $374 \pm 17$ & $323 \pm 33$ & $308 \pm 26^{* *}$ & $201 \pm 76$ \\
\hline \multicolumn{8}{|l|}{ Striatum } \\
\hline salinc & $137 \pm$ & $74 \pm 05$ & $5531 \pm 628$ & $1331 \pm 126$ & $798 \pm 141$ & $353+26$ & $212 \pm 33$ \\
\hline PEA & $124 \pm 37$ & $76 \pm 05$ & $5415 \pm 473$ & $1499 \pm 154$ & $776 \pm 194$ & $342 \pm 21$ & $220 \pm 82$ \\
\hline
\end{tabular}

Rats were decapitated 4 weeks after PEA withdrawal, and the contents of brain amines and their metabolites were measured by HPLC-ECD. Results are shown as the mean \pm S.E.M. (ng/g tissue) from 6 rats. Statistical differences: ${ }^{*} \mathrm{P}<0.05,{ }^{* *} \mathrm{P}<0.01$ (Student's $t$-test).

\section{Contents of brain amines and their metabolites}

Significant increases in NA levels in the cerebral cortex and significant decreases in 5HT levels in the hypothalamus were found (Table 1). However, DA, DOPAC and HVA levels showed no significant changes.

\section{DISCUSSION}

We have demonstrated that reverse tolerance to stereotyped behavior was induced after repeated PEA treatment. This is consistent with previous reports by other authors $(5,7$, 8).

PEA is known to induce an amphetaminelike release of DA from presynaptic neurons and an inhibition of DA re-uptake $(2,22,23)$. The previous tissue studies $(24,25)$ suggest that the presynaptic response to PEA of the striatal DA nerve terminals is augmented in rats with repeated PEA treatment. Moreover, results from the direct measurement of extracellular levels of DA in rats using in vivo brain microdialysis (8) or push-pull cannula (23) indicate that the augmented behavioral response after repeated PEA treatment may be explained by enhanced DA release as observed after repeated AMPH $(9,25,26)$ or MAP treatment $(11,12)$. On the other hand,
PEA is also reported to exert a direct stimulating effect on postsynaptic dopamine receptors (27) or its own specific binding site (28). The details of the related mechanism still remain to be studied.

It is interesting that the levels of the striatal DA, DOPAC and HVA did not change long after withdrawal from PEA treatment (Table 1), although some neurochemical changes are proposed in the striatal DA nerve terminals.

It is known that there are differences in the behavioral effects between PEA and MAP. The hyporesponsiveness to acoustic stimulation that had been observed after withdrawal from repeated MAP treatment (10) was not found after withdrawal from PEA. However, in this study, reverse tolerance to stereotyped behavior was induced after repeated PEA treatment as observed after withdrawal from MAP $(10-12)$. This suggests that the responsiveness to acoustic stimulation is irrelevant to the induction of the reverse tolerance. Moreover, neurochemically, significant decreases in 5-HT and 5-HIAA levels in the cerebral cortex, which had been observed after withdrawal from MAP (10), were not found after PEA withdrawal.

Significant increases in NA levels in the cerebral cortex were found 4 wecks after PEA 
withdrawal. However, there are no reports that have investigated the levels of NA or MHPG in the brain long after withdrawal from PEA treatment. The details of the related mechanism still remain to be studied.

PEA is an endogenous trace amine and known to play an important physiological role in the brain (1). In this study, we used a relatively high dose $(50 \mathrm{mg} / \mathrm{kg})$ of PEA. We need to use a lower dose of PEA with type B monoamine oxidase inhibitor to study the details of the effects of PEA on the related mechanisms in the brain.

In conclusion, it may be suggested that the response to acoustic stimulation after repeated PEA administration in rats can not be a model for the abnormal responsiveness to environmental stimulation that is observed in chronic paranoid schizophrenics.

\section{REFERENCES}

1 Nakajima, T., Kamimoto, Y. and Sano, I.: Formation of $\beta$-phenylethylamine in mammalian tissue and its effect on motor activity in the mouse. J. Pharmacol. Exp. Ther. 143, 319-325 (1964)

2 Motohashi, N., Nakagawara, M., Semba, J., Ishii, K., Watanabe, A. and Kariya, T.: Effect of $\beta$ phenylethylamine on locomotor activity and brain catecholamine metabolism in mice. Japan. J. Psychopharmacol. 3, 67-75 (1983)

3 Cole, S.O.: Brain mechanisms of amphetamine-induced anorexia, locomotion, and stereotypy: a review. Neurosci. Biobehav. Rev. 2, 89-100 (1978)

4 Dourish, C.T.: Local application of $\beta$-phenylethylamine to the caudate nucleus of the rat elicits locomotor stimulation. Pharmacol. Biochem. Behav. 22, $159-162$ (1985)

5 Borison, R.L., Havdala, H.S. and Diamond, B.I.: Chronic phenylethylamine stereotypy in rats: A new animal model for schizophrenia? Life Sci. 21, $117-122(1977)$

6 Dourish, C.T. and Boulton, C.T.: The effects of acute and chronic administration of beta-phenylethylamine on food intake and body weight in rats. Prog. Pharmacol, 5, $411-414$ (1981)

7 Borison, R.L. and Diamond, B.I.: A new animal model for schizophrenia: Interaction with adrenergic mechanisms. Biol. Psychiatry 13, 217 225 (1978)
8 Kuroki, T., Tsutsumi, T., Hirano, M., Matsumoto, T., Tatebayashi, Y., Nishiyama, K., Uchimura, H.. Shiraishi, A., Nakahara, T. and Nakamura, K.: Behavioral sensitization to beta-phenylethylamine (PEA): enduring modifications of specific dopaminergic neuron systems in the rat. Psychopharmacology 102, 5-10 (1990)

9 Robinson, T.E. and Becker, J.B.: Enduring changes in brain and behavior produced by chronic amphetamine administration: a review and evaluation of animal models of amphetamine psychosis. Brain Res. Rev. 11, 157-198 (1986)

10 Akita, H., Hashimoto, M., Yamada, M., Kiuchi, Y., Oguchi, K. and Yasuhara, H.: Behavioral characteristics associated with acoustic stimulation and the neurochemical alterations of monoaminergic systems in rat brain at the steady state of repeated methamphetamine administration. Folia Pharmacol. Japon. 95, 327 - 333 (1990) (Abs. in English)

11 Ichikawa, J.: Change of behavior and central monoaminergic systems in the rat after repeated methamphetamine pretreatment: Presynaptic regulatory mechanism. Japan. J. Psychopharmacol. 8, $389-403$ (1988)

12 Nishikawa, T., Mataga, N., Takashima, M. and Toru, M.: Behavioral sensitization and relative hyperresponsiveness of striatal and limbic dopaminergic ncurons after repeated methamphetamine treatment. Eur. J. Pharmacol. 88, 195-203 (1983)

13 Kokkinidis, L. and Anisman, H.: Amphetamine models of paranoid schizophrenia: an overview and elaboration of animal experiment. Psychol. Bull. 88, $551-579$ (1980)

14 Bell, D.S.: The experimental reproduction of amphetamine psychosis. Arch. Gen. Psychiatry 29. $35-40$ (1973)

15 Mckinney, W.T. and Moran, E.C.: Animal models of schizophrenia. Am. J. Psychiatry 13, 478-483 (1981)

16 Williams, M.: Psychophysiological responsiveness to psychological stress in early chronic schizophrenic reactions. Psychosom. Med. 15, 456-462 (1953)

17 Zahn, T.P.: Autonomic nervous system characteristics possibly related to a genetic predisposition to schizophrenia. Schizophrenia Bull. 3, 49-60 (1977)

18 Matsubara, K. and Matsushita, A.: Changes in ambulatory activities and muscle relaxation in rats after repeated doses of diazepam. Psychopharmacology 77, 279-283 (1982)

19 Kilts, C.D., Breese, G.R. and Mailman, R.B.: Simultancous quantification of dopamine, 5-hy- 
droxytryptamine and four metabolically related compounds by means of reversed-phase highperformance liquid chromatography with electrochemical detection. J. Chromatogr. 225, 347-357 (1981)

20 Warnhoff, M.: Simultaneous determination of norepinephrine, dopamine, 5-hydroxytryptamine and their main metabolites in rat brain using high performance liquid chromatography with electrochemical detection. J. Chromatogr. 307, 271281 (1984)

21 Glowinski, J. and Iversen, L.L.: Regional studies of catecholamines in the rat brain- $\mathrm{I}$. The disposition of $\left[{ }^{3} \mathrm{H}\right]$ norepinephrine, $\left[{ }^{3} \mathrm{H}\right]$ dopamine and $\left[{ }^{3} \mathrm{H}\right.$ ]dopa in various regions of the brain. $\mathrm{J}$. Neurochem. 13, 655-669 (1966)

22 Ishibashi, K., Matsumoto, T., Hirano, M., Hirano, H., Nakamura, K., Nakahara, T. and Uchimura, $\mathrm{H}_{\text {: }} \beta$-Phenylethylamine (PEA)-induced behavioral sensitization and changes in monoamine metabolism in discrete rat brain regions. Neurochem. Res. 11, $1799-1800$ (1986)

23 Bailey, B.A., Philips, S.R. and Boulton, A.A.: In vivo release of endogenous dopamine, 5-hydroxy- tryptamine and some of their metabolites from rat caudate nucleus by phenylethylamine. Neurochem. Res. 12, 173-178 (1987)

24 Nakagawara. M.: $\beta$-Phenylethylamine; psychopharmacology and clinical aspects. Japan. J. Psychopharmacol. 6, 295-307 (1986)

25 Muller, P. and Seeman, P.: Presynaptic subsensitivity as a possible basis for sensitization by longterm dopamine mimetics. Eur. J. Pharmacol. 55, $149-157$ (1979)

26 Robinson, T.E., Jurson, P.A., Bennett, J.A. and Bentgen, K.M.: Persistent sensitization of dopamine neurotransmission in ventral striatum (nucleus accumbens) produced by prior experience with (+)-amphetamine: a microdialysis study in freely moving rats. Brain Res. 462, 211-222 (1988)

27 Antelman, S.M., Edwards, D.J, and Lin, M.: Phenylethylamine: evidence for a direct, postsynaptic dopamine-receptor stimulating action. Brain Res. 127, 317-322 (1977)

28 Hauger, R.L., Skolnick, P. and Paul, S.M.: Specific $\left[{ }^{3} \mathbf{H}\right]$-phenylethylamine binding sites in rat brain. Eur. J. Pharmacol. 83, 147-148 (1982) 